\title{
Different generation regimes of mode-locked all-positive-dispersion all-fiber Yb laser
}

\author{
Sergey Kobtsev ${ }^{\mathrm{a}}$, Sergey Kukarin ${ }^{\mathrm{a}}$, Sergey Smirnov ${ }^{\mathrm{a}}$, Sergey Turitsyn ${ }^{\mathrm{b}}$, Anton Latkin ${ }^{\mathrm{a}}$ \\ ${ }^{a}$ Laser system laboratory, Novosibirsk State University, Pirogova 2, Novosibirsk, 630090 Russia; \\ ${ }^{\mathrm{b}}$ Aston University, Birmingham, B4 7ET, UK
}

\begin{abstract}
Different generation modes of all-positive-dispersion all-fibre $\mathrm{Yb}$ laser mode-locked due to effect of non-linear polarization evolution are investigated. For the first time we realized in the same laser both generation of single picoseconds pulse train and a newly observed lasing regime where generated are picosecond wave-packets, each being a train of femtosecond sub-pulses. Using both experimental results and numerical modeling we discuss in detail the mechanisms of laser mode-locking and switching of generation regimes and show a strong dependence of output laser characteristics on configuration of polarization controllers. A good qualitative agreement between experimental results and numerical modeling is demonstrated.
\end{abstract}

Keywords: femtosecond lasers, picoseconds lasers, nonlinear polarization evolution, dissipative solitons.

\section{INTRODUCTION}

Nonlinear polarisation evolution (NPE) $)^{1-4}$ is a commonly employed mechanism used to achieve stable mode-locking and ultra-short pulse generation in an all-positive dispersion cavity. In comparison to systems using semiconductor saturable absorbers, the NPE-based lasers are relatively easier to assemble and they are more robust against power damage. In addition, NPE-based laser systems allow simple adjustment of the saturable absorber action, and as a result, they can be effectively used for studies of different pulse generation regimes. Compared to soliton generation in the anomalous group velocity dispersion (GVD) regime, use of the normal dispersion allows one to achieve higher pulse energies without wave breaking ${ }^{4-6}$. The key to achieving stable generation of high energy pulses is the management of nonlinear propagation effects in the cavity. Another promising approach to high energy pulse generation in fiber lasers introduced earlier ${ }^{7}$ is to elongate the laser cavity in order to achieve extremely low pulse repetition rates with considerable increase in pulse energy. Applying this technique we have recently demonstrated the generation of highly chirped short pulses with few-nanosecond width and energy as high as $3.9 \mu \mathrm{J}^{7}$ and $4 \mu \mathrm{J}^{8}$ both in a hybrid (consisting of both bulk elements and fiber sections) and all-fiber lasers with optical length of the cavity amounting to 3.8 and $8 \mathrm{~km}$, respectively. Modelocked fiber laser generating high energy pulses typically presents a multi-parametric nonlinear system that by changing device parameters might operate in a range of regimes: conventional soliton, dispersion-managed soliton (stretched pulse), self-similar pulse regime, all-normal dispersion highly-chirped pulse, multiple-pulse regimes and so on. In this work we present in more detail an unusual mode of operation of all-positive dispersion fiber lasers and demonstrate how a change of system parameters leads to switching between standard stable pulse train generation and more exotic regimes. In particular, we study both experimentally and numerically a generation of a double-scale picosecond lump envelope and a femtosecond scale noise-like oscillations inside the larger wave-packet. Such a lasing mode is characterised by an unusual double-feature shape with an auto-correlation function (ACF) comprising of a femtosecondscale peak on a picosecond-scale pedestal. Though noise-like pulse generation regime has been studied for Er-doped fiber lasers ${ }^{9-12}$, the nature of fine structure oscillations is not yet completely clear. Thus some authors attribute the noiselike pulse generation to large birefringence of the laser cavity in a combination with the nonlinear field evolution ${ }^{10}$. However a similar noise-like pulse operation was demonstrated in erbium-doped fiber laser with weak birefringence ${ }^{11}$. It was conjectured ${ }^{11}$ that the formation of the noise-like oscillations is caused by the pulse collapse effect ${ }^{13}$ and that such dynamics is a generic feature of a range of the passively mode-locking systems. The pulse collapse effect studied ${ }^{13}$ is an intermediate dynamical process that is caused by the third-order nonlinear gain term in the Taylor expansion of the

Fiber Lasers VII: Technology, Systems, and Applications, edited by Kanishka Tankala, Jay W. Dawson, Proc. of SPIE Vol. 7580,758028 - @ 2010 SPIE · CCC code: 0277-786X/10/\$18 · doi: 10.1117/12.841237 
nonlinear response of the effective saturable absorber. For some range of parameters, initially smooth field distribution is engaged in an explosive-like growth of the amplitude with corresponding compression. The collapse effect dynamics is more sensitive to initial conditions and as a result the forming field structures tend to be more random and complex compared to non-collapse pulse generation regimes. Though, evidently such fast growth of the field amplitude is saturated at some level by the higher terms in the expansion and other high-order effects, the resulting noisy and random asymptotic state could be quite different from more smooth regimes of the radiation intensity growth. Here we present experimental and numerical studies of the double-scale femto-pico-second optical lumps generation in $\mathrm{Yb}$ fiber laser and discuss in detail physical mechanisms behind the generation of mode switching in mode-locked all-positive-dispersion fiber lasers. In addition to recent work ${ }^{14}$ we present here our new results of adiabatic cavity elongation as well as analytical description of switching generation regimes.

\section{Experimental setup and numerical modeling}

The experimental set-up of the considered all-fiber ring-cavity laser is shown in Fig. 1. A 7-m long active ytterbium fiber with a $7-\mu \mathrm{m}$ core was used as the laser active element. This fiber was pumped from one of the ends of the twinned fiber (GTWave technology ${ }^{15}$ ) with $1.5 \mathrm{~W}$ of 980 -nm radiation from a laser diode. In order to couple the generated radiation out of the resonant cavity, a fiber-based polarisation beam splitter (FPBS) was used. In order to ensure uni-directional generation, a polarisation-independent fiber optical isolator (PIFI) was also introduced into the cavity. Mode locking was achieved due to the effect of non-linear polarisation evolution ${ }^{1,2,3}$. For control of the polarisation state in the layout, two fiber-based polarisation controllers PC1 and PC2 were employed.

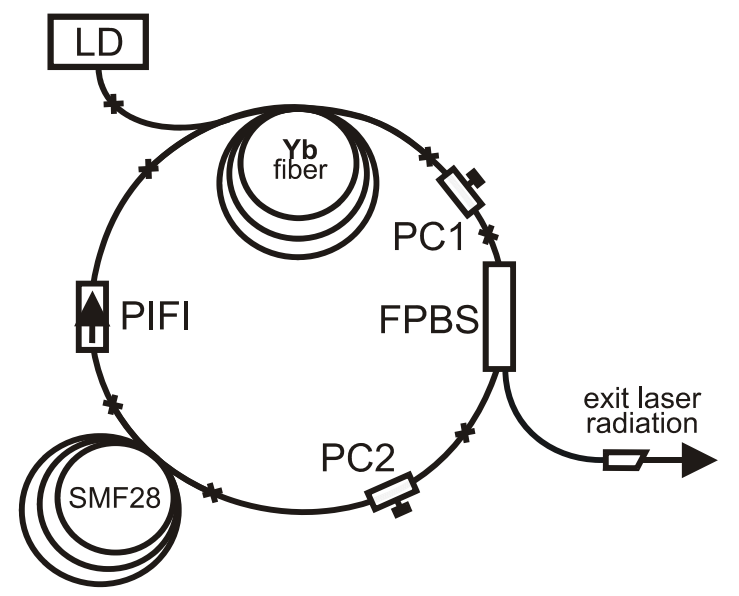

Fig. 1. Experimental laser scheme: PC - polarization controller, PIFI - polarization-independent fiber isolator, FPBS - fiber polarization beam splitter, LD - pump laser diode.

For elongation of the laser cavity and boosting of the output pulse energy a stretch of passive SMF-28 fiber was inserted. Using pieces of SMF of different lengths one can change considerably repetition rate and energy of generated pulses. All optical fibers in this layout had normal dispersion within the working spectral range of the laser. The average output power of the laser was limited by the power rating of FPBS and did not exceed $150 \mathrm{~mW}$.

In the theoretical analysis of operation of considered laser schemes a standard approach is to apply numerical modelling based on the system of coupled modified non-linear Schrödinger equations ${ }^{16}$ :

$$
\begin{aligned}
& \frac{\partial A_{x}}{\partial z}=i \gamma\left\{\left|A_{x}\right|^{2} A_{x}+\frac{2}{3}\left|A_{y}\right|^{2} A_{x}+\frac{1}{3} A_{y}^{2} A_{x}^{*}\right\}+\frac{g_{0} / 2}{1+E /\left(P_{\text {sat }} \cdot \tau\right)} A_{x}-\frac{i}{2} \beta_{2} \cdot \frac{\partial^{2} A_{x}}{\partial t^{2}}, \\
& \frac{\partial A_{y}}{\partial z}=i \gamma\left\{\left|A_{y}\right|^{2} A_{y}+\frac{2}{3}\left|A_{x}\right|^{2} A_{y}+\frac{1}{3} A_{x}^{2} A_{y}^{*}\right\}+\frac{g_{0} / 2}{1+E /\left(P_{s a t} \cdot \tau\right)} A_{y}-\frac{i}{2} \beta_{2} \cdot \frac{\partial^{2} A_{y}}{\partial t^{2}}
\end{aligned}
$$


where $A_{x}, A_{y}$ are the orthogonal components of the field envelope, $z$ is a longitudinal co-ordinate, $t-$ time, $\gamma=4.7 \times 10^{-5}$ $(\mathrm{cm} \cdot \mathrm{W})^{-1}-$ non-linear coefficient, $g_{0}-$ unsaturated gain coefficient, $\beta_{2}=23 \mathrm{ps}^{2} / \mathrm{km}-$ dispersion coefficient, $P_{\text {sat }}-$ saturation power for the active fiber, $\tau$ - time of cavity round trip. In our calculations we neglected the higher-order dispersion and linear bi-refringence of the fiber. The amplifier parameters were estimated from experimental measurements and taken equal to $\mathrm{g}_{0}=540 \mathrm{~dB} / \mathrm{km}, \mathrm{P}_{\text {sat }}=52 \mathrm{~mW}$. To improve convergence of the solution to the limiting cycle, the numerical modelling included a spectral filter with a $30-\mathrm{nm}$ band-width, which exceeds considerably the typical width of generated laser pulses. The effect of polarisation controllers was taken into account by introduction of corresponding unitary matrices (see e.g. recent publication ${ }^{17}$ and references therein). For simplification of the analysis and reduction of the required computation resources we shortened the laser cavity to $6 \mathrm{~m}$ in our calculations. Furthermore we only varied three of the 6 possible polarisation controller parameters $(\alpha, \beta, \psi)$ using matrices $(2,3)$ for $\mathrm{PC} 1,2$ at $\varphi=2 \psi+\pi / 4$ :

$$
\begin{aligned}
P C_{1} & =\left(\begin{array}{cc}
\cos \alpha & -i \cdot \sin \alpha \\
-i \cdot \sin \alpha & \cos \alpha
\end{array}\right) \\
P C_{2} & =\left(\begin{array}{cc}
\cos \beta-i \sin \beta \cos 2 \varphi & -i \sin \beta \sin 2 \varphi \\
-i \sin \beta \sin 2 \varphi & \cos \beta+i \sin \beta \cos 2 \varphi
\end{array}\right) \cdot(-i) \cdot\left(\begin{array}{cc}
\cos 2 \psi & \sin 2 \psi \\
\sin 2 \psi & -\cos 2 \psi
\end{array}\right)
\end{aligned}
$$

In spite of simplifications we made the modelling allowed us to reproduce qualitatively the experimental results.

\section{Stable pulse generation}

One of the features of discussed laser mode locked due to NPE is a variety of generation regimes available at different settings of polarization elements. Thus in our recent experiments ${ }^{7,8}$ we were able to achieve a mode-lock using a large variety of ways by re-adjusting polarization controllers. Laser pulses generated at different PC settings have different energy and duration.

In order to have a deeper insight into our previous experimental observations we reproduced these results in numerical simulations as described above. In order to reproduce generation of laser pulses with different energy and duration we varied polarisation controller parameters $(\alpha, \beta, \psi)$ in a random way. For each set of PC angles we performed numerical modelling starting with a noise-like intra-cavity field distribution and simulating a large number of round-trips through the cavity. About $5 \%$ of our simulations ended with a stable mode-lock, i.e. we observed a bell-shaped intensity distribution with no changes after any whole number of subsequent radiation round-trips through the cavity. As a result we were able to obtain a large number of points in three-dimensional space of angles $(\alpha, \beta, \psi)$ where each point corresponds to stable mode-lock with its own pulse parameters. Analyzing these points shown in Fig. 2 in a E-T diagram we can see considerable change in pulse energy and duration caused by re-adjustment of polarization controllers what agrees with our previous experimental observations.

In order to fulfil more detailed comparison we plotted experimental and simulated spectra and auto-correlation traces in Fig. 3. A good qualitative agreement between modeling and experiments can be clearly seen. Both experimental and simulated spectra have steep spectral edges (see Fig. 3 a,c) whereas intensity ACFs are bell-shaped (see Fig. 3 b,d) indicating that a train of single pulses is generated. Since the cavity has all-positive dispersion, the generated laser pulses exhibit large chirp ${ }^{4-6}$ and may be efficiently compressed with an external diffraction-grating compressor, what is confirmed by both the numerical results and the experiment.

\section{Noise-like generation}

An interesting feature of considered all-normal-dispersion laser is possibility of different types of operation. Thus in addition to aforementioned stable pulse generation regime the laser permits the second type of operation where an unusual double-structured ACF (femto- and pico-second) can be observed, see Fig. 4 b, e. Experimentally measured laser generation spectra of this type usually has a smooth bell-shaped appearance (see Fig. 4 a). However, as the numerical simulation demonstrates, such smooth spectra are a result of averaging over a very large number of pulses, whereas the spectrum of an individual pulse contains an irregular set of noisy-like peaks (see a non-averaged spectrum in Fig. $4 \mathrm{~d}$ shown with a grey line). In the temporal representation this type of generation corresponds to a picosecond wave packet 


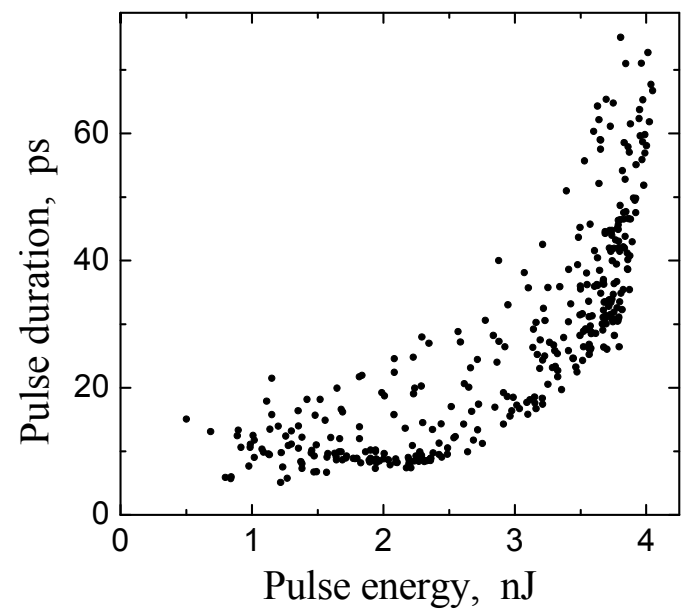

Fig. 2. Numerical simulations of stable single-pulse generation regime. Each point represents duration and energy of pulse obtained in simulations carried out with certain parameters $\left(\alpha_{k}, \beta_{k}, \psi_{k}\right)$ of polarization controllers.
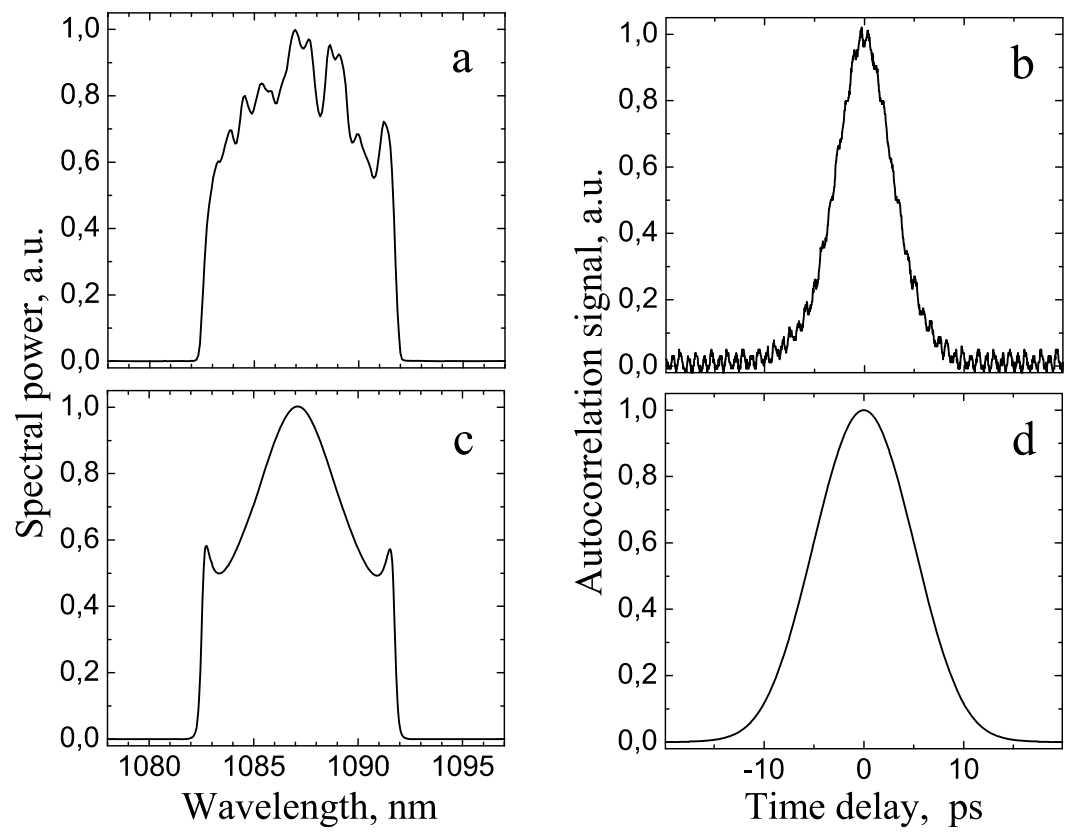

Fig. 3. Stable single-pulse generation: a,b - experimental results, c,d - simulations; a,c spectra, $b, d$ - intensity auto-correlation functions.

consisting of an irregular train of femtosecond sub-pulses (see Fig. $4 \mathrm{f}$ ). Peak power and width of such sub-pulses stochastically changes from one round trip along the cavity to another, also leading to fluctuations in the wave packet parameters usually easily noticeable when observing the output pulse train in real time on the oscilloscope screen during experiments (see Fig. $3 \mathrm{c}$ ). We do not observe any systematic change in generation parameters such as power or wavepacket duration even after several hours of generation. The absence of systematic drift of pulse parameters is typical for numerical simulations as well (in the latter case however much shorter pulse train of about only $5 \times 10^{4}$ was examined.) 

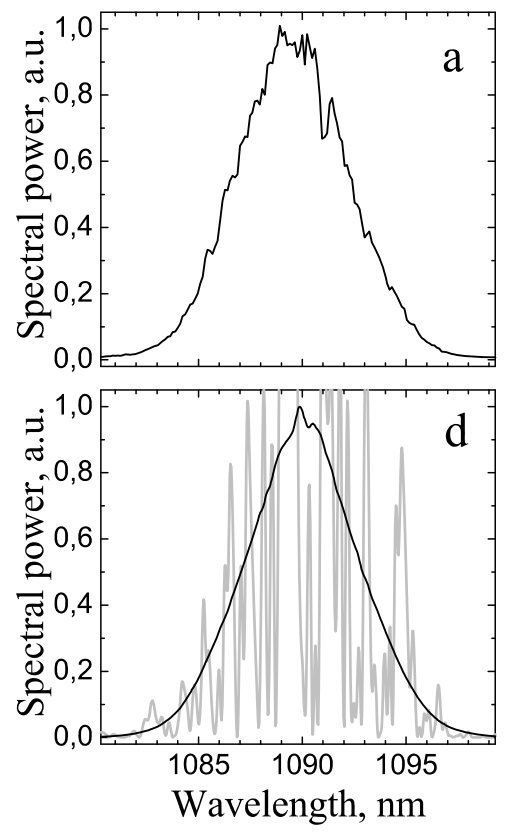

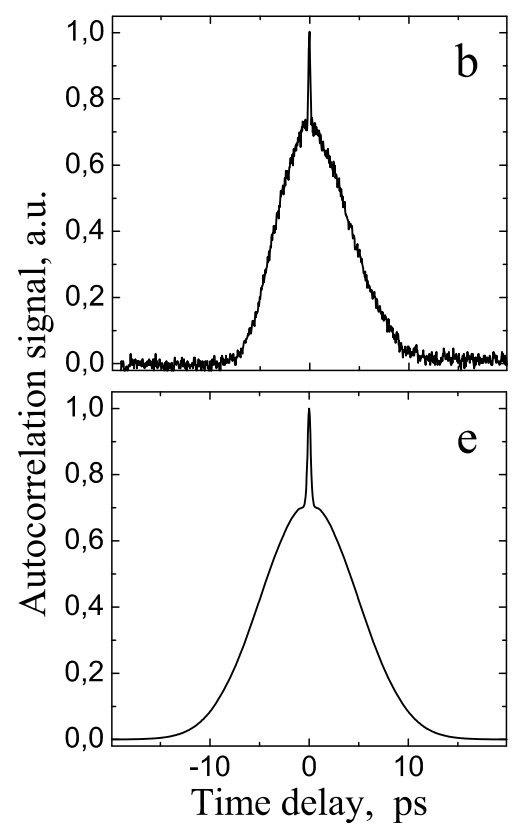

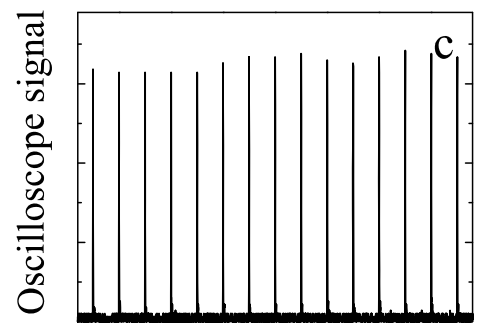

Time, a.u.

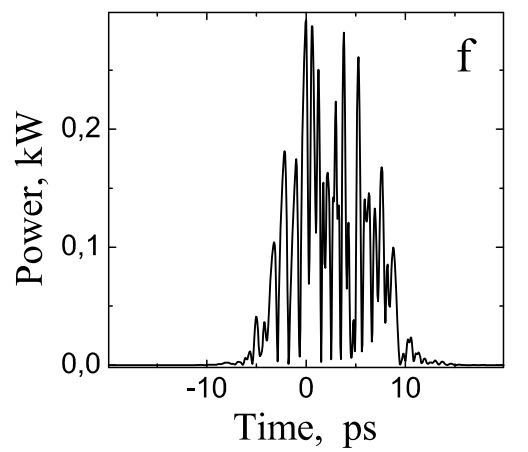

Fig. 4. Noise-like generation regime: a-c - experimental results, d-f - simulations; a,d -spectra, b,e - ACFs, c - pulse train from oscilloscope, $\mathrm{f}$ - non-averaged intensity distribution.

The discussed regime presents an interesting symbiotic co-existence of stable solitary wave dynamics and low-dimension stochastic oscillations. Note that similar structures have been studied in different context numerically in the complex cubic-quintic Ginzburg-Landau model in [17]. The co-existence of stable steady state pulses and pulsating periodic, quasi-periodic or stochastic localised structures is a general feature of multi-parametric dissipative nonlinear system. Each particular nonlinear dynamic regime exists in a certain region of parameter space. Therefore, in systems that possess a possibility to switch operation from one region of parameters to another, one can observe very different lasing regimes. The regions of parameters where pulsating (periodic, quasi-periodic or stochastic) localized structures do exist might be comparable or even larger than regions of existence of conventional steady state solitons. Note that in the multidimensional space of parameters of the considered laser scheme, it is almost impossible to explore all of operational regimes via direct modelling.

Both experiment and simulation show that wave-packets generated in the double-scale femto-picosecond regime can be compressed only slightly and pulses after compression remain far from spectrally limited. As our experiments have demonstrated, after extra-cavity compression of these complex wave packets with the help of two diffraction gratings ACF of the resulting pulses has qualitatively the same double-feature shape.

\section{Elongation of laser cavity and switching of generation regimes}

Both stable pulse generation and noise-like regime can be easily obtained in experiments and numerical simulations by re-adjusting settings of intra-cavity polarization elements such as polarization controllers or half- and quarter-wave plates when a cavity length is quite short (about several meters). However when we increase the length of laser cavity up to dozens or hundreds of meters in our modeling and perform the similar procedure of re-adjustment PC settings in a random way we find that for longer cavities probability of stable pulse generation dramatically decreases. This fact is illustrated in Fig. 5 where shown is the number of points in three-dimensional space of PC parameters $(\alpha, \beta, \psi)$ that we found in the course of numerical simulations.

In order to have insight into switching of lasing regimes we performed adiabatic cavity elongation starting with a point $(\alpha, \beta, \psi)$ that corresponds to stable single pulse generation. Then we gradually elongated laser cavity increasing its length by $\sim 1 \%$. After each act of cavity elongation we carried out numerical modeling of large number $(\mathrm{N} \sim 50)$ round-trips with fixed parameters in order to achieve steady-state laser generation. Performing this procedure repeatedly 
we investigated laser generation in adiabatically elongated cavity. In particular we obtained dependence of laser output pulses energy on cavity length (see Fig. 6). It's clearly seen that the initial stage of cavity elongation corresponds to smooth linear growth of pulse energy due to reduction of laser repetition rate and consequent increase of saturation energy of the active fiber. However at certain point energy dependence in Fig. 6 experiences a jump after which a smooth linear growth changes into stochastic-like curve. The latter preserves linear trend in the course of following cavity elongation despite clearly visible fluctuations.

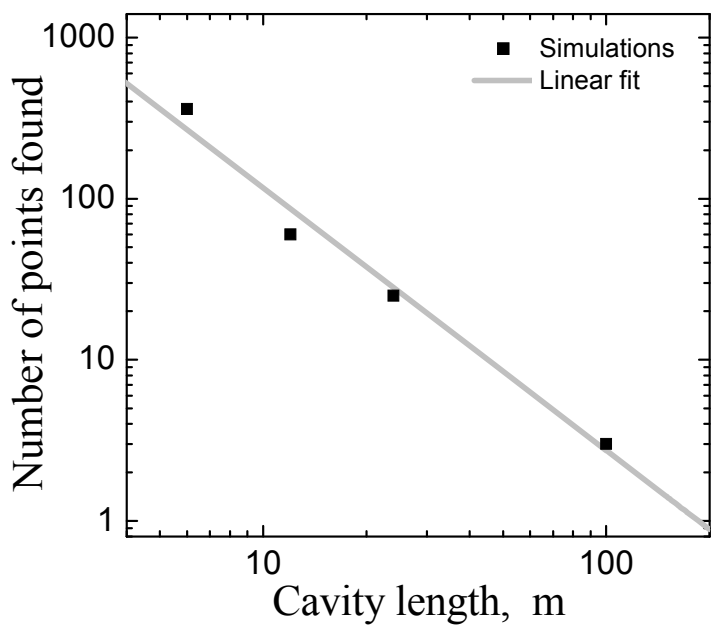

Fig. 5. Number of points found in numerical simulations in threedimensional space of PC angles $(\alpha, \beta, \psi)$ which correspond to stable single-pulse generation in cavities of different lengths.

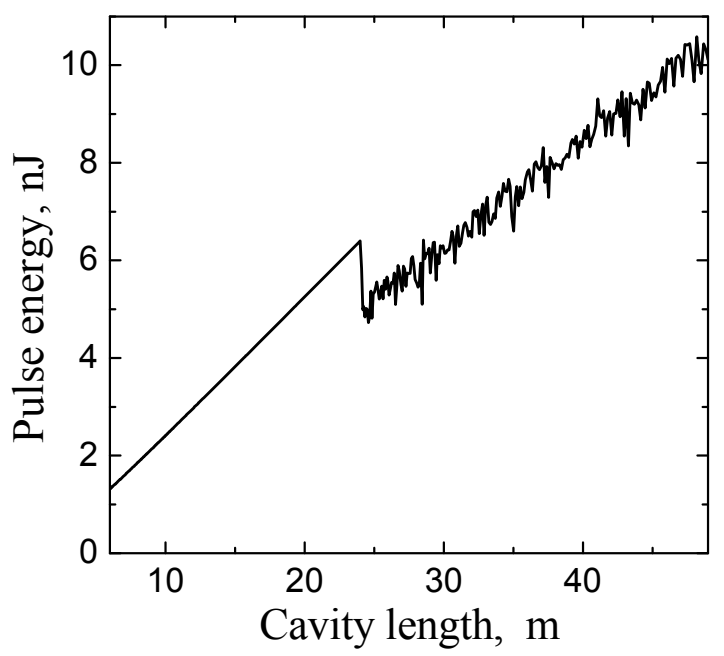

Fig. 6. Increase of laser output pulses energy in the course of numerical simulations of adiabatic cavity elongation.

Analysis of temporal intensity distributions, spectra and pulse-to-pulse fluctuations just before and right after the critical point in Fig. 6 reveals that the change in energy dependence corresponds to switching of lasing regimes. Indeed, just before the critical point the laser generates bell-shaped pulses with spectra having typical steep edges similar those shown in Fig. 3. After passing the critical point in the course of cavity elongation the bell-shaped pulse becomes unstable and decays into a series of noise-like sub-pulses (see Fig. 4). The spectra exhibit noisy and peaky structure as 
well, and after ensemble average they become bell-shaped with no steep edges typical for stable single-pulse generation regime.

As we could see the switching between generation regimes is accompanied with development of some instability that leads to break-up of single pulse into a stochastic series of noise-like sub-pulses. In order to reveal the origin of this instability we performed additional numerical modeling and investigated the dependence of net gain per roundtrip on pulse power. In particular, we simulated propagation of intra-cavity laser pulses with "scaled" power through the cavity. When scale factor is equal to unity the pulse parameters do not change after a whole cavity round-trip since the pulse a steady solution of propagation equations. In contrast, when scale factor is not equal to unity the pulse is not a solution and therefore it changes after cavity round-trip. The ratio between pulse energy after and before the roundtrip is the pulse net gain at first round-trip. The net gain can be easily found in simulations and it includes saturated gain in Yb-fiber as well as energy losses at spectral filter and PBS.

Dependence of net gain on scale factor obtained in numerical modeling is shown in Fig. 7 for three different lengths of laser cavity. For stable single-pulse generation both pulse power scale factor and net gain at first round-trip equal to unity. It's essential that the slope of net gain curve is negative in the vicinity of the point of stable generation. However the same graph contains an interval of pulse powers that correspond to negative slope of net gain (denoted as area of instability in Fig. 7). Once the laser enters this interval no stable pulse generation is possible as any growth in pulse power is further amplified and finally the pulse break-up occurs. As can be seen in Fig. 7 when one increases the cavity length the width of area where stable generation is possible decreases. As a result even extremely small fluctuations of pulse power can bring the laser into the area of instability what leads to pulse break-up and switching into noise-like generation regime.

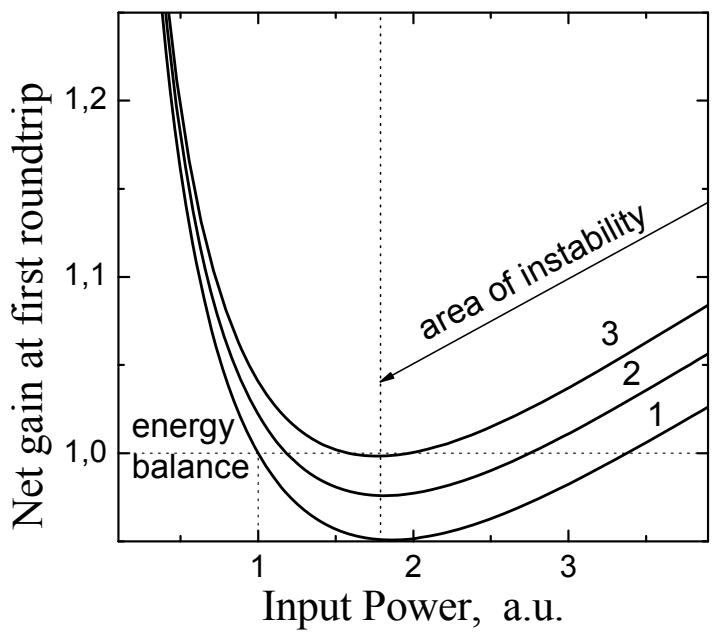

Fig. 7. Dependence of net gain per roundtrip vs. initial pulse power obtained in numerical modeling. Curves 1-3 correspond to slightly different cavity cavity lengths close to boundary of single-pulse generation regime area $(\mathrm{L}=12.0,12.275$ and $12.55 \mathrm{~m}$ for curves 1,2 and 3 .)

Virtually the same dependence of net gain at the first round-trip on pulse power can be obtained in analytical description of laser generation based on the same set of coupled NLSE (1). Although NLSE can't be integrated in general case, as our numerical modeling shows, for typical laser parameters dispersive terms can be neglected in first approximation as well as spectral filter. This allows us to integrate NLSE (1) both for SMF and active fiber in terms of elementary and special functions and therefore to match each optical element with a two-dimensional matrix (its elements depend in particular on intra-cavity pulse power in the case of optical fibers). In particular, stretch of active fiber corresponds to

$$
\hat{A}=\left(\begin{array}{cc}
\cos \Omega & -\sin \Omega \\
\sin \Omega & \cos \Omega
\end{array}\right) \cdot \sqrt{\frac{E_{\text {sat }}}{E_{\text {in }}} \cdot f^{-1}\left\{f\left(\frac{E_{\text {in }}}{E_{\text {sat }}}\right)+g_{0} z\right\}} \cdot \exp \left(i \varphi_{N L}\right)
$$


where $f(x)=x+\ln x, E_{\text {in }}$ - pulse energy at the fiber input, $E_{\text {sat }}=P_{\text {sat }} \times \tau$ - saturation energy, $z-$ fiber length, $\varphi_{N L}-$ inessential non-linear phase shift, and the angle of polarization rotation is given by (5):

$$
\Omega(z)=-\left.\frac{2}{3} \frac{\gamma J}{E_{\text {in }}} \cdot \frac{E_{\text {sat }}}{g_{0}} \cdot\left(\frac{x^{2}}{2}+x\right)\right|_{A} ^{B}, \quad A=\frac{E_{\text {in }}}{E_{\text {sat }}}, \quad B=f^{-1}\left\{f\left(\frac{E_{\text {in }}}{E_{\text {sat }}}\right)+g_{0} z\right\}
$$

Passing to the limit $g_{0} \rightarrow 0$ one can obtain similar expression for passive fiber. Once we have matrices for each optical element, electrical field evolution after a whole round-trip can be easily described using a product of these matrices. It allows us to find intra-cavity laser pulse peak power and energy after a single round-trip as a function of input pulse power. The ratio between pulse energy after and before the round-trip is depicted in Fig. 8. One can see that this dependence agrees well with that shown in Fig. 7 obtained in numerical modeling. Let's note that the dependence in Fig. 8 was obtained for slightly different lengths of laser cavity, however the difference between the lengths used in numerical simulations and analytical description is less than $4 \%$. Thus there is both qualitative and quantitative agreement between full-numerical simulations and simplified analytical description which takes into account only nonlinear terms of NLSE.

Our analytical description of stable single-pulse generation gives deeper inside into switching of lasing regimes. Thus when one increases cavity length towards critical point pulse peak power found as a root of equation $\mathrm{g}=1$ (net gain is equal to unity) is increased (see Fig. 8), what agrees well with our numerical simulations. However when the critical point is reached the root vanishes, i.e. for any pulse peak power net gain $\mathrm{g}>1$. It means that no stable single-pulse generation is possible and the laser switches to noise-like generation regime.

Let's note that the same conclusions remain valid if one changes PC settings instead of the length of laser cavity. In this case similar dependences of net gain can be obtained both in numerical simulations and analytical description.

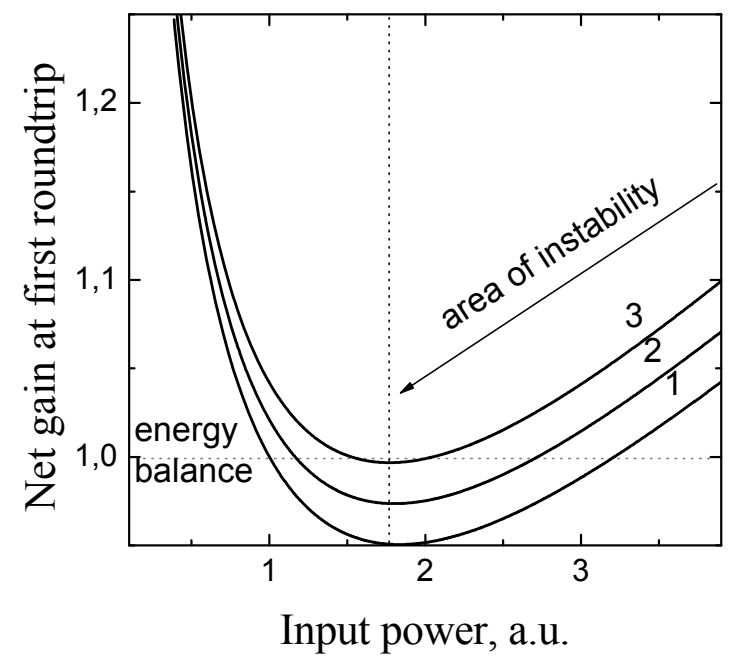

Fig. 8. Dependence of net gain per roundtrip vs. initial pulse power obtained analytically. Curves 1-3 correspond to slightly different cavity cavity lengths close to boundary of singlepulse generation regime area $(\mathrm{L}=11.55,11.8$ and $12.05 \mathrm{~m}$ for curves 1,2 and 3.)

\section{CONCLUSIONS}

To the best of our knowledge we present for the first time a noise-like generation regime in ytterbium fiber laser with allpositive dispersion and mode locking due to non-linear polarisation evolution. Both the experimental and numerical studies show an unusual feature typical of this new generation type that is a double-structured (pico- and femto-second) 
non-collinear ACF (intensity ACF). Applying both extensive numerical modelling and analytical description we have shown that physical mechanism underlying the new generation regime is an instability leading to decomposition of isolated pico-second pulses into a stochastic sequence of femtosecond sub-pulses. This instability originates purely from transmission function of PBS and optical fibers that turn polarization ellipse of intra-cavity laser pulse. This effect makes it quite difficult to generate regular trains of isolated ultra-short pulses in long-resonator mode-locked all-positivedispersion fiber lasers based on non-linear polarization evolution effect and very careful adjustment of the system parameters is required in this case. On the other hand, pico-second scale optical lumps with femto-second oscillatory structures and broadband spectrum might be attractive for a range of practical applications.

\section{ACKNOWLEDGEMENTS}

This work was supported by a Grant "Scientific and educational staff of innovational Russia in 2009-2013" (Grant No П2490).

\section{REFERENCES}

[1] Tamura, K., Haus, H. A., and Ippen, E. P., "Self-starting additive pulse mode-locked erbium fiber ring laser," Electron. Lett. 28, 2226-2228 (1992).

[2] Chen, C. J., Wai, P.K.A., and Menyuk, C. R., "Soliton fiber ring laser," Opt. Lett. 17, 417-419 (1992).

[3] Matsas, V.J., Newson, T. P., Richardson, D. J., and Payne, D. N., "Selfstarting passively mode-locked fiber ring soliton laser exploiting non linear polarisation rotation," Electron. Lett. 28, 2226-2228 (1992).

[4] Chong, A., Renninger, W.H., and Wise, F.W., "Propeties of normal-dispersion femtosecond fiber lasers," J. Opt. Soc. Am. B 25, 140-148 (2008).

[5] Wise, F.W., Chong, A., and Renninger, W.H., "High-energy femtosecond fiber lasers based on pulse propagation at normal dispersion," Laser \& Photonics Reviews , 2, 58-73, (2008).

[6] Kalashnikov, V.L., Podivilov, E., Chernykh, A., and Apolonski, A., "Chirped-pulse oscillators: theory and experiment," Appl. Phys. B 83, 503-510 (2006).

[7] Kobtsev, S., Kukarin, S., and Fedotov, Yu., "Ultra-low repetition rate mode-locked fiber laser with high-energy pulses," Optics Express 16, 21936-21941 (2008).

[8] Kobtsev, S., Kukarin, S., Smirnov, S., Latkin, A., and Turitsyn, S., "High-energy all-fiber all-positive-dispersion mode-locked ring $\mathrm{Yb}$ laser with $8 \mathrm{~km}$ optical cavity length," CLEO-Europe/EQEC-2009, CJ8.4. Munich, Germany, 1419 June 2009.

[9] Horowitz, M., Barad, Y., and Silberberg, Y., "Noiselike pulses with a broadband spectrum generated from an erbium-doped fiber laser," Opt. Lett. 22 (11), 799-801 (1997).

[10] Horowitz, M., and Silberberg, Y., "Control of noiselike pulse generation in erbium-doped fiber lasers," IEEE Phot. Technol. Lett. 10, 1389-1391 (1998).

[11] Zhao, L.M., Tang, D.Y., Wu, J., Fu, X.Q., and Wen, S.C., "Noise-like pulse in a gain-guided soliton fiber laser," Optics Express 15, 2145-2150 (2006).

[12] Chouli, S., and Grelu, Ph., "Rains of solitons in a fiber laser," Optics Express 17, 11776-11781 (2009).

[13] Chernykh, A.I., and Turitsyn, S.K., "Soliton and collapse regimes of pulse generation in passively modelocking laser systems," Opt. Lett. 20, 398-400 (1995).

[14] Kobtsev, S., Kukarin, S., Smirnov, S., Turitsyn, S., and Latkin, A., "Generation of double-scale femto/pico-second optical lumps in mode-locked fiber lasers," Optics Express 17, 20707-20713 (2009).

[15] Grudinin, A.B., Payne, D.N., Turner, P.W., Nilsson, L.J.A., Zervas, M.N., Ibsen, M. and Durkin, M.K., "Multi-fiber arrangements for high power fiber lasers and amplifiers," United States Patent 6826335, 30.11.2004.

[16] Agrawal, G.P., [Nonlinear fiber optics], Academic press, 2001.

[17] Komarov, A., Leblond, H., and Sanchez, F., "Quintic complex Ginzburg-Landau model for ring fiber lasers," Phys. Rev. E 72, 025604 (2005).

[18] Akhmediev, N., Soto-Crespo, J. M., and Town, G., "Pulsating solitons, chaotic solitons, period doubling, and pulse coexistence in mode-locked lasers: Complex Ginzburg-Landau equation approach," Phys. Rev. E, 63, 056602 (2001). 